\title{
A cohesin-independent mechanism modulates recombination activity along meiotic chromosomes
}

Jonna Heldrich ${ }^{1}$, Tovah E. Markowitz ${ }^{1, \#}$, Luis A. Vale-Silva ${ }^{1}$, and Andreas Hochwagen ${ }^{1, *}$

${ }^{1}$ Department of Biology, New York University; New York, NY 10003; USA

\# Current address: Leidos Biomedical Research, Inc.; Bethesda, MD; USA

* Correspondence: andi@nyu.edu

Running title: Cohesin-independent recruitment of meiotic axis proteins 


\section{Summary}

Meiotic chromosomes organize around a cohesin-dependent axial element, which promotes meiotic recombination and fertility. In the absence of cohesin, axial-element proteins instead accumulate in poorly understood genomic regions. Here, we show in S. cerevisiae that these regions are particularly enriched for axis proteins even on wild-type chromosomes and thus reflect a cohesin-independent recruitment mechanism. By contrast, other organizers of chromosome structure, including cohesin, condensin, and topoisomerases, are depleted from the same regions. This spatial patterning is observable before meiotic entry and therefore independent of meiotic recombination. Indeed, the regional density of gene-coding sequences is sufficient to predict a large fraction of cohesin-independent axis protein binding, suggesting that the gene-associated chromatin landscape plays a role in modulating axis protein deposition. The increased accumulation of axis proteins in these regions corresponds to a greater potential for initiation of recombination and progression to crossovers.

Key words: Synaptonemal complex, Rec8, Red1, Hop1, DSB hotspot, nucleosomes, Rtf1 


\section{Introduction}

Inheritance of genetic information in sexually reproducing organisms depends on a reductional cell division called meiosis [1-3]. This process involves the dangerous yet necessary infliction of DNA double-strand breaks (DSBs), which lead to exchange of DNA between homologous chromosomes during meiotic recombination. Meiotic DSBs occur within the framework of a complex 3D organization of the genome, which promotes compaction, individualization, pairing and repair of chromosomes in preparation for chromosome segregation during the first of the two meiotic divisions. Chromosomes fold into loops that are anchored together at their bases by the axial element, a proteinaceous scaffold made up of meiosis-specific axis proteins that, in the budding yeast Saccharomyces cerevisiae, include Red1, Hop1, and Rec8-cohesin $[4,5]$. The axial element provides binding sites for accessory factors essential for DSB formation and is required for both normal levels and normal distribution of meiotic DSBs [6-8].

The placement of axial-element proteins is largely determined by cohesin, which localizes axis proteins to discrete sites along the chromosomes and finetunes their binding to gene ends $[6,7]$. In the absence of Rec8, no axis structure is detectable by electron microscopy and loop formation is disrupted $[9,10]$. In yeast and plant rec8 mutants, axis proteins nevertheless continue to bind chromosomes, albeit at reduced levels and in an aberrant pattern $[6,7,11,12]$

(Figure 1A-C). In these mutants, DSB formation is generally restricted to regions that retain axis protein binding $[6,7,12]$. Here, we show that this retention of axis proteins reflects a Rec8independent recruitment mechanism for axis proteins that is also active during wild-type meiosis. We show that this patterning is independent of meiotic entry, that regional gene density is sufficient to predict a substantial fraction of axis-protein distribution, and that the regional enrichment of axis proteins is associated with greater levels of DSB and crossover activity on wild-type chromosomes. 


\section{Results}

\section{Definition of regions of axis-protein enrichment}

To define the regions associated with axis-protein enrichment bioinformatically, we reanalyzed ChIP-seq data of the yeast axis component Red1 in a rec8 8 mutant by dividing the genome into windows and setting an enrichment threshold (see Methods). Windows with Red1 signal above the threshold were joined with immediately adjacent windows of significant Red1 signal, and these genomic regions were termed clusters (Figure 1C). The intervening regions were defined as deserts.

As shown previously [6,7], clusters are distributed in a nonuniform pattern across the 16 yeast chromosomes (Figure 1C). The size, number, and relative positions of clusters vary for each chromosome and show no obvious association with centromeres or telomeres. One consistent pattern that distinguishes the small chromosomes (I, III, VI) is their almost complete coverage by clusters, while the large chromosomes are overwhelmingly made up of deserts.

\section{Differential enrichment of core axis factors along wild-type chromosomes}

We wondered whether the biased binding of Red1 in clusters also occurs on wild-type chromosomes. We analyzed existing ChIP-seq data of axis factors obtained from wild-type yeast at 3 hours after meiotic induction $[7,13]$, the time when meiotic DNA breakage is maximal [14]. This analysis showed that even in wild-type cells, Red1 binds significantly more in cluster regions than in deserts (Figure 1B, D and Figure S1A).

One reason for this differential enrichment could be the fact that axis attachment sites, defined as peaks of enrichment of the axis proteins Red1, Hop1, and Rec8, are more densely spaced in clusters (Figure 1E). However, axis-protein binding levels at these sites do not support this hypothesis. Whereas Red1 and Hop1 were indeed significantly more enriched at axis sites 
located in cluster regions (Figure 1H-I), Rec8 was more enriched at axis sites in deserts

(Figure 1J). Another component of the cohesin complex, Scc2, bound with the same trends as

Rec8 (Figure 1K). The complementary enrichment of Red1/Hop1 and cohesin implies the existence of a second recruitment mechanism that recruits Red1 and Hop1 to wild-type chromosomes without cohesin.

In line with this interpretation, the extra enrichment of Red1 and Hop1 in clusters is not spatially connected to cohesin-dependent enrichment peaks. This additive effect was particularly obvious when analyzing Red1 enrichment across gene bodies. Metagene analysis showed a uniform increase of Red1 binding in the cluster regions overlaid over the biased recruitment to gene ends mediated by Rec8 (Figure 1F-G). These data indicate that the second recruitment mechanism creates broad domains of enrichment and acts in parallel to the focal axis-protein enrichment mediated by Rec8-cohesin.

In the absence of Rec8, chromosomal association of Red1 becomes fully dependent on Hop1 [7], raising the possibility that Hop1 helps mediate cohesin-independent recruitment of Red1 on wild-type chromosomes. Indeed, analysis of Red1 enrichment in a hop $1 \Delta$ mutant showed Red1 enriched in deserts rather than clusters (Figure 1L), matching the pattern of Rec8 (Figure 1J). This outcome is in keeping with the notion that Red1 binding becomes fully dependent on cohesin in the absence of Hop1 [7]. We conclude that Hop1 is responsible for cohesinindependent recruitment of Red1.

\section{Differential enrichment in clusters and deserts does not require meiotic entry}

Further analysis showed that three additional regulators of meiotic chromosome structure, condensin, topoisomerase I (Top1) and topoisomerase II (Top2), are also differentially distributed between these regions on wild-type chromosomes. Analysis of available ChIP-seq 
data of PK9-tagged Smc4, a subunit of condensin [13], Top1-13myc, and Top2 [15] showed significantly greater binding of all three proteins around axis sites in deserts compared to clusters (Figure 1M-O). Desert enrichment of Top1 and Top2 was even more apparent at DSB hotspots (Figure S1B-C), which often overlap with peaks of topoisomerase enrichment [15]. Notably, the biased enrichment of Top2 in deserts was also observed in the absence of meiotic DSBs (spo11-YF and spo11A) (Figure S1D-E). Indeed, topoisomerases and condensin were enriched in deserts even in premeiotic or cycling cells (Figure S1F-H). These data indicate that the signals differentiating clusters and deserts are not linked to the meiotic program.

Mutant analysis did not reveal obvious roles for condensin or Top2 in defining clusters and deserts. Conditional nuclear depletion of the condensin subunit Brn1 by anchor-away [16] caused vegetative cell lethality [17] but did not alter the relative enrichment of Red1 in cluster regions (Figure S1I-J). Similarly, inactivation of TOP2, using the temperature-sensitive top2-1 allele at $34^{\circ} \mathrm{C}[15,18]$, had no effect on the greater relative enrichment of Red1 and Hop1 in clusters (Figure S1K-N). Although we cannot exclude redundant effects, our data indicate that neither cohesin, nor condensin or Top2 alone are responsible for differentiating cluster and desert regions.

\section{Clusters are associated with longer genes and increased coding density}

The apparent independence from the meiotic program prompted us to investigate whether inherent chromosomal features could explain the formation of clusters and deserts. Previous analysis of Red1 enrichment in rec8 mutants had indicated that long genes are significantly over-enriched in regions of Red1 binding [7]. Consistent with this analysis, we found that the median length of open reading frames in clusters was $17.6 \%$ greater than in deserts (clusters = 1433 bp; deserts = 1218 bp) (Figure 2A). By contrast, deserts exhibited significantly larger intergenic regions (Figure 2B). The greatest difference was observed for divergent regions, 
which had an $85.6 \%$ ( 279 bp) larger median size in deserts than in clusters, but the same trend was also observed for tandem regions (39.4\% or $\sim 124$ bp larger in deserts) and convergent regions $(29.4 \%$ or $\sim 55 \mathrm{bp}$ larger in deserts). These data indicate that clusters are associated with both increased gene size and increased coding density.

The median sizes of clusters and deserts are approximately $15 \mathrm{~kb}$ and $22.5 \mathrm{~kb}$, which correspond to around 9 and 14 genes, respectively, and raises the possibility that cluster formation may be a regional effect. Indeed, logistic fit analysis after segmenting the genome into 5-kb bins showed a strong bias toward high coding density (coding nucleotides/total DNA) in bins defined as clusters (Figure 2C).

To probe the significance of this regional association in explaining axis protein recruitment, we trained a logistic regression model, using $80 \%$ of the genome as a learning set for predicting clusters and deserts in the remaining $20 \%$ based on coding density. This approach showed that nearly $70 \%$ of the test set could be accurately predicted as either a cluster or a desert based on the regional coding density alone (coding nucleotides/total DNA per 5kb window) (Figure 2D). This number improved only marginally to $72.5 \%$ when we excluded the short chromosomes (Figure S2A-B), indicating that known cis-encoded features that increase recombination activity on short chromosomes [19-21] have only minor impact on the overall performance of this model. Thus, regional coding density is a major predictor of axis-protein cluster formation.

We also observed a substantial effect of cluster size on Red1 enrichment. When clusters were separated into 3 quantiles by length, average Red1 enrichment per bp scaled significantly with cluster length (based on 95\% C.I.; Figure 2E). This association implies that larger cluster regions are better able to recruit or retain Red1 binding. 


\section{Nucleosome occupancy and order is elevated in clusters}

We speculated that the higher coding density of clusters could be manifested in a different overall chromatin state, as genes in the S. cerevisiae genome are typically associated with wellordered nucleosomes [22]. Therefore, we used published data to analyze nucleosome enrichment patterns in clusters and deserts. Analysis of the ChIP-seq signal of histones H3 [23] and $\mathrm{H} 4$ [24] during meiotic prophase showed a significant enrichment of both histones in cluster regions (Figure 3A-B). Similar results were observed after micrococcal nuclease digestion (MNase-seq) of meiotic prophase samples [15,25] (Figure 3C-D), as well as prior to meiotic entry (Oh; Figure S3A). Clusters also exhibited a more pronounced signal periodicity (Figure 3C), indicating that nucleosomes are more highly ordered than in deserts. This higher spatial order is also apparent when specifically analyzing MNase-seq patterns around meiotic DSB hotspots (Figure 3D).

We investigated whether differences in nucleosome stability or periodicity could underlie the formation of clusters and deserts. The acetylation of histone $\mathrm{H} 4$ on lysine 44 (H4K44ac) regulates nucleosome stability in meiosis and has been implicated in meiotic DSB activity [24]. Analysis of existing ChIP-seq data showed an enrichment of H4K44ac around hotspots located in clusters (Figure 3E), but this enrichment was largely explained by the higher nucleosome levels in these regions (Figure 3F) and is also seen for $\mathrm{H} 3 \mathrm{~K} 4 \mathrm{me}$, another hotspot-associated histone modification (Figure 3G-H). However, MNase-seq data showed that the differential enrichment of nucleosomes around hotspots in cluster regions persisted in a non-acetylatable H4K44R mutant (Figure S3B-C). Thus, H4K44ac-associated nucleosome turnover cannot explain the different levels of nucleosomes in clusters versus deserts.

To probe the role of nucleosome order in establishing clusters and deserts, we analyzed mutants lacking the PAF1C subunit Rtf1, which has been implicated in nucleosome positioning 
[26]. MNase-seq analysis of $r t f 1 \Delta$ mutants revealed reduced nucleosome order along genes, as indicated by the less pronounced periodicity of nucleosome peaks in clusters relative to the +1 nucleosome (Figure S3D-E). However, the relative enrichment of Red1 in clusters was unaffected by the absence of RTF1 (Figure S3F), indicating that Red1 binding in clusters is not affected by altered nucleosome order. Accordingly, Red1 enrichment in clusters was also unaffected by the loss of Set1 and Dot1, two histone methyltransferases that are regulated by PAF1C in meiosis and promote Red1 binding $[11,27]$ (Figure S3G-H). Together, these analyses exclude several known modulators of meiotic chromatin states as regulators of cluster and desert formation.

\section{Meiotic recombination activity is more frequent in clusters}

As Red1 and Hop1 are necessary to support wild-type levels of meiotic DSB formation and recombination, we asked whether the differential enrichment of axis proteins had functional consequences for meiotic recombination along wild-type chromosomes. As expected from the narrower nucleosome-free regions in clusters (Figure 3D), average Spo11-oligo levels, which report on meiotic DSB distribution $[25,28]$ showed a more focused peak for hotspots located in clusters compared to desert hotspots (Figure 4A-C). In addition, average Spo11 activity per bp was elevated in cluster hotspots (Figure 4C), suggesting more concentrated DSB formation. Indeed, the area under the Spo11-oligo curve was mildly but significantly greater at cluster hotspots (Figure S4A), even though the density of hotspots did not differ between clusters and deserts (Figure S4B), indicating that hotspots in cluster regions have on average a higher likelihood of initiating meiotic recombination. Consistent with higher DSB activity, sequences surrounding cluster hotspots also saw a greater enrichment of histone $\mathrm{H} 2 \mathrm{~A}$ phosphorylated on serine $129(\mathrm{pH} 2 \mathrm{~A})$, an indicator of meiotic DSB activity (Figure 4D-E), although this enrichment is partly driven by the greater histone occupancy in clusters (Figure S4C). 
We asked whether the higher enrichment of axis proteins in clusters also impacts the chance of crossover repair in these regions. The SUMO ligase Zip3 governs the designation of DSBs for crossover repair and is a cytological and genomic marker of crossover designation [29-31]. Indeed, analysis of published Zip3 ChIP-seq data [31] indicated a greater enrichment of Zip3 at cluster hotspots (Figure 4F-G). These data imply that cohesin-independent enrichment of axis proteins leads to regions of increased recombination activity on wild-type chromosomes.

\section{Discussion}

Here, we show that the previously observed regional enrichment of axis proteins in yeast rec8 mutants $[6,7]$ reflects a second, cohesin-independent mechanism for axis-protein recruitment that is active in wild-type cells and impacts the frequency of meiotic recombination activity along meiotic chromosomes. Similar cohesin-independent association of axis proteins is also observed in Arabidopsis thaliana [12], suggesting that this mechanism may be conserved.

Cytological experiments in yeast have revealed chromosomal domains of differential axis protein enrichment that also have a higher chance of exhibiting Zip3 foci [32-34]. Therefore, it is tempting to speculate that the cluster and deserts defined here reflect the domains defined by cytology. The uniform enrichment of axis proteins in cluster regions, although mild overall, could indeed be broad enough to be cytologically observable. Moreover, axis protein-rich domains alternate with regions enriched for Rec8-cohesin by cytology [34], in line with our analyses. One argument against a direct correspondence is that enrichment of Hop1 in clusters is unchanged in mutants lacking the chromosome remodeler Pch2 (Figure S10), whereas the cytological domains disappear in pch2 mutants (Börner et al., 2008), but this could be an effect of higher Hop1 signal masking the underlying structure. 
The differential enrichment between clusters and deserts is also seen for several other chromosome structure factors, including condensin and topoisomerases, implying that these regions exhibit fundamental differences in chromosome or chromatin organization. Cohesin, condensin, and topoisomerases are all enzymes that can alter chromosome structure [35-38], suggesting that deserts are structurally more active than cluster regions. However, our analyses found no evidence that this structural activity displaces axis proteins from deserts. Alternatively, structural activity could come from processes such as transcription, because genes in clusters have on average lower steady-state mRNA levels (16.1\% smaller median) than genes in deserts during early prophase (Figure S4D). Arguing against this possibility is the fact that the transcriptional program changes dramatically as cells enter the meiotic program $[15,39,40]$, yet clusters and deserts can be detected even prior to meiotic entry by the differential enrichment of nucleosomes, topoisomerases, and condensin. We therefore favor the interpretation that the differential distribution of chromosome structure factors is a secondary consequence of inherent properties of the chromatin fiber.

The notion that the features differentiating clusters and deserts are to a large extent hard-wired into the local chromatin environment is supported by our finding that regional coding density can predict approximately $70 \%$ of the cluster and desert regions. The fact that the elevated axis protein enrichment in clusters occurs uniformly across gene bodies suggests that the feature recruiting axis proteins may be relatively broad and non-specific. One such feature that clearly differentiates clusters is the higher density and order of nucleosomes. However, altering nucleosome order did not affect Red1 binding in clusters, arguing against a role for this feature in recruiting axis proteins. We also excluded roles for two histone methyltransferases, Set1 and Dot1, which are required for wild-type levels of Red1 recruitment [11]. It is possible that other chromatin marks or even inherent nucleosomal features, such as unmodified histone surfaces or the associated DNA conformations, could create a broad signal that modulates binding of 
axis proteins, but this question requires further investigation. Intriguingly, the axis factor Hop1, which is required for Red1 recruitment to cluster regions ([7] and this study), binds structured DNA in vitro $[41,42]$. Thus, a better understanding of Hop1 in vivo chromatin binding activities may help identify the chromatin features differentiating clusters from deserts.

Ultimately, the cohesin-independent enrichment of axis proteins has consequences for meiotic recombination. Clusters exhibit elevated levels of meiotic DSB formation and DNA damage signaling, and indeed are the only sequences that show substantial levels of meiotic DSB formation in rec8 mutants [6-8]. Importantly, clusters also exhibit significantly higher levels of the crossover marker Zip3, which is essential for regularly spaced crossover formation $[30,31,43]$. Thus, our data identify a novel layer of crossover regulation that modulates the patterns of recombination along meiotic chromosomes.

\section{Acknowledgements}

We thank N. Hollingsworth for sharing antibodies, G. Vader for helpful discussions, and the NYU Department of Biology Sequencing Core for technical assistance and data processing. This work was supported by the National Institutes of Health [GM111715 and GM123035 to $\mathrm{AH}]$.

\section{Author contributions}

Conceptualization, J.H., T.E.M. and A.H.; Investigation, J.H., T.E.M., L.A.V.S., and A.H.; Software, J.H.; Formal analysis, J.H., T.E.M. and A.H.; Resources, J.H., T.E.M. and A.H.; Writing - Original Draft, J.H and A.H.; Writing - Review \& Editing, J.H., T.E.M., L.A.V.S., and A.H.

\section{Declaration of Interests}

The authors declare no competing interests. 
bioRxiv preprint doi: https://doi.org/10.1101/2020.08.11.247122; this version posted August 12, 2020. The copyright holder for this preprint (which was not certified by peer review) is the author/funder, who has granted bioRxiv a license to display the preprint in perpetuity. It is made

B

VI suldewidull

III Antwithen

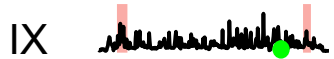

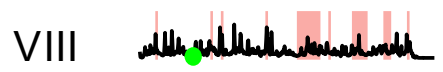

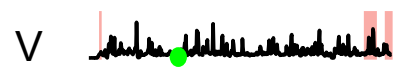

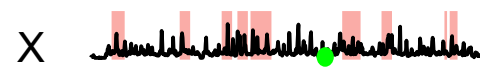

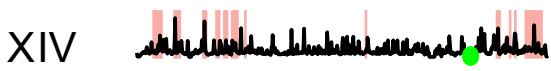

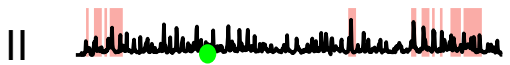

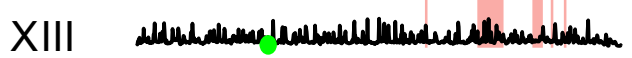

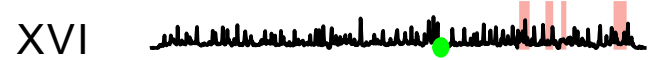

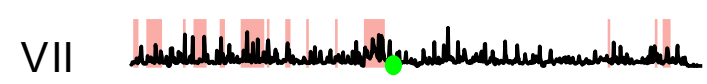

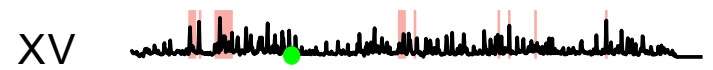

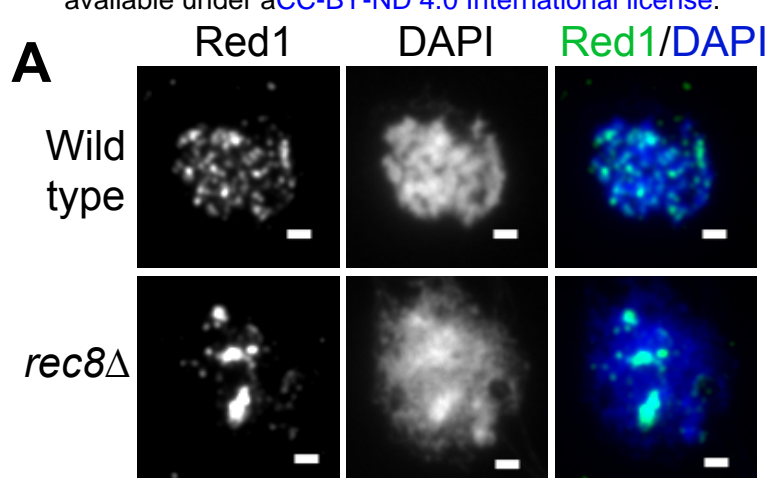

C whith I Anuman VI Herentill III
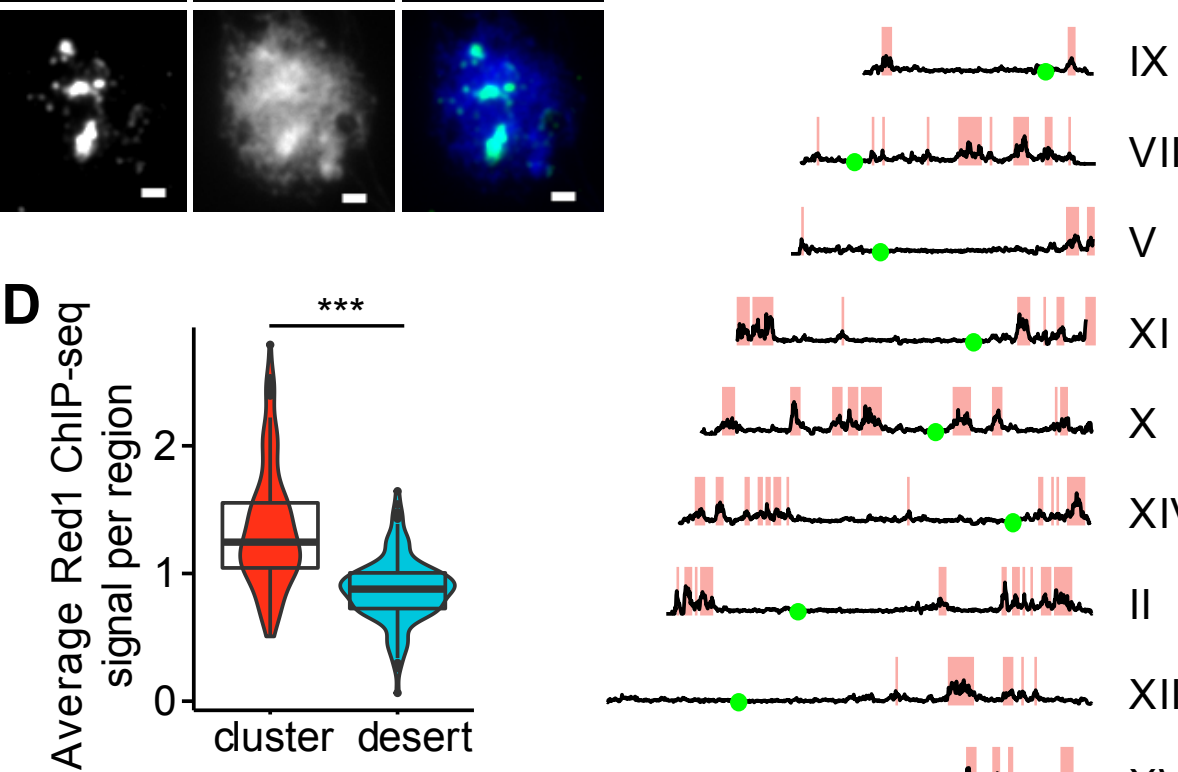

Mn

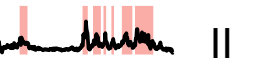

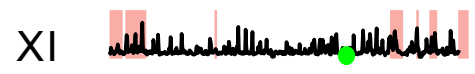

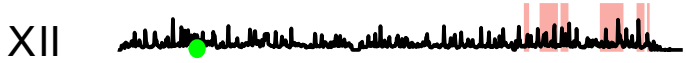
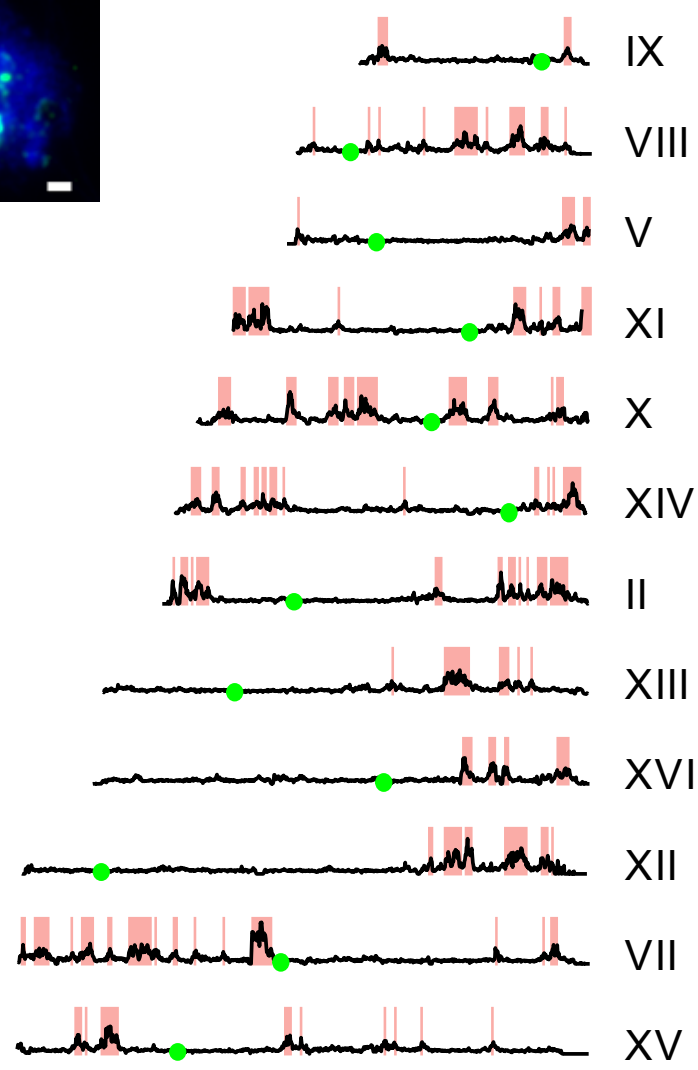

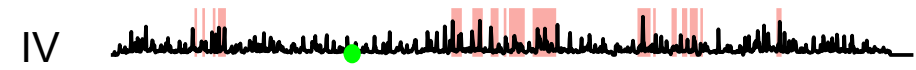
- Red1 signal

E
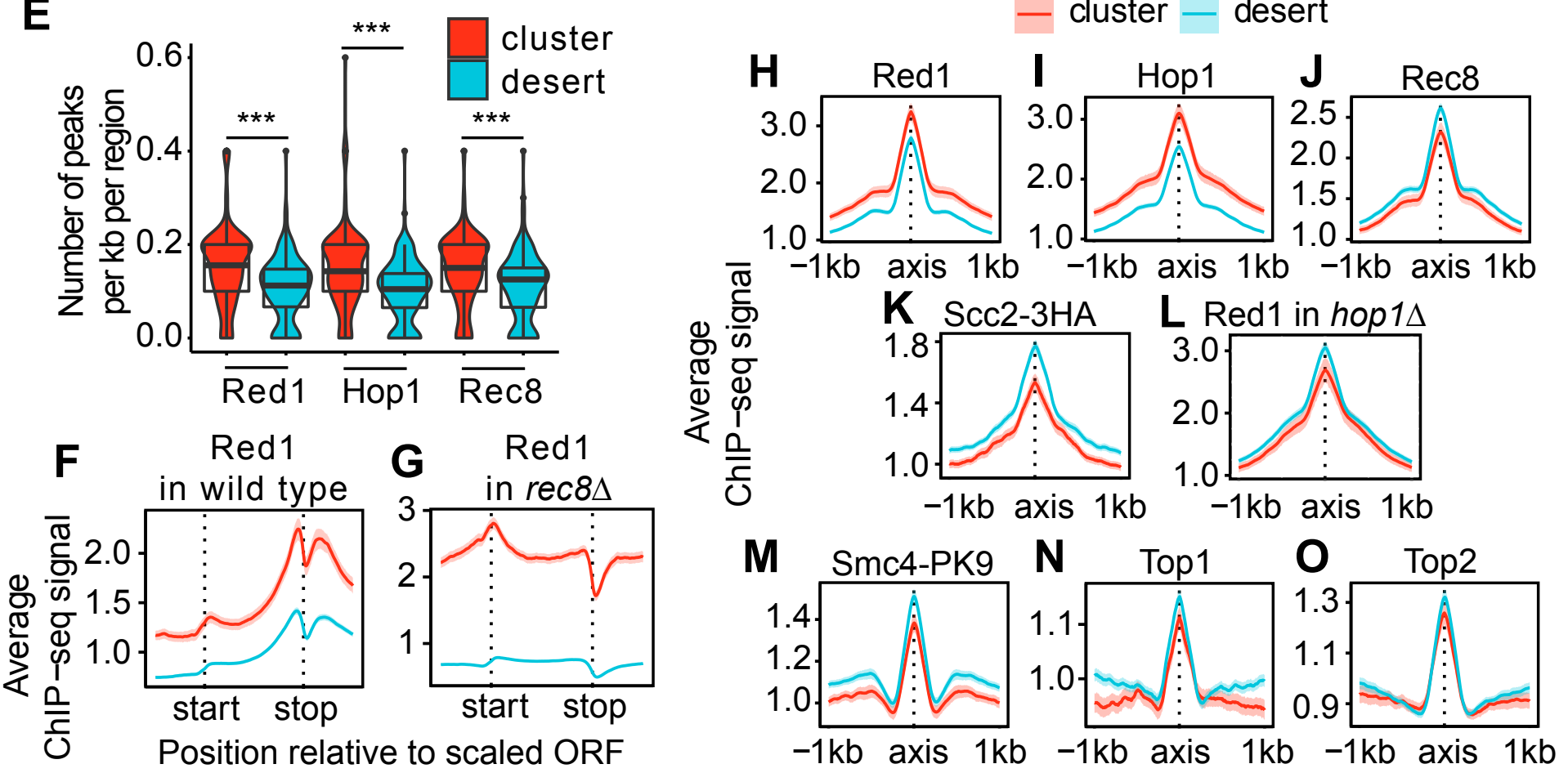


\section{Figure Legends}

Figure 1. Cohesin-independent recruitment of axial elements boosts Red1 binding in wild type.

(A) Representative images of immunofluorescence staining for Red1 (green) and DAPI (blue) on chromosome spreads of wild-type and rec8s mutant cells 5 hours after meiotic induction. Bar, $1 \mu \mathrm{m}$. (B) Wild-type and (C) rec8 $\Delta$ genomic distribution of Red1 (black) on all chromosomes at the time of DSB formation (3 hours after meiotic induction) as measured by ChIP-seq. Red underlay delineates regions designated as clusters. Green dots mark the centromeres. (D) Violin and box plots quantifying the average Red1 signal per cluster or desert region on wild-type chromosomes. ${ }^{* *} P<0.0001, t$-test. (E) Quantification of the density of Red1, Hop1, and Rec8 peaks per kb of cluster (red) and desert (blue) regions. ${ }^{* * *} P<0.0001$, Mann-Whitney-Wilcoxon test. Metagene analysis of Red1 in wild type $(F)$ and $\operatorname{rec} 8 \Delta(G)$ indicating average ChIP-seq enrichment along genes in clusters and deserts. Average Red1 $(\mathrm{H})$, Hop1 (I), Rec8 (J), Scc2-3HA (K), Red1 in hop1 $(\mathrm{L})$, Smc4-PK9 (M), Top1 (N), and Top2 (O) binding in wild type at axis attachment sites [13] in clusters and deserts. The $95 \%$ confidence interval for the average lines is shown for H-O. See also Figure S1. 
bioRxiv preprint doi: https://doi.org/101101/2020 08 11247122. this version posted August 122020 . The copyright holder for this preprint (which was not certified by peer review) is the author/funder, who has granted bioRxiv a license to display the preprint in perpetuity. It is made available under aCC-BY-ND 4.0 International license.

A

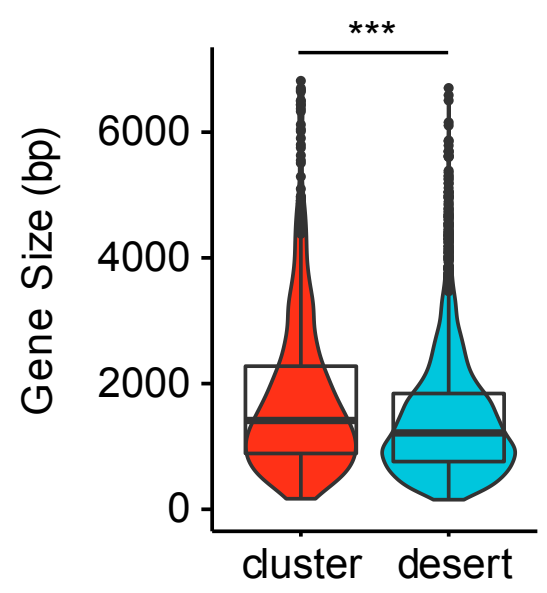

B

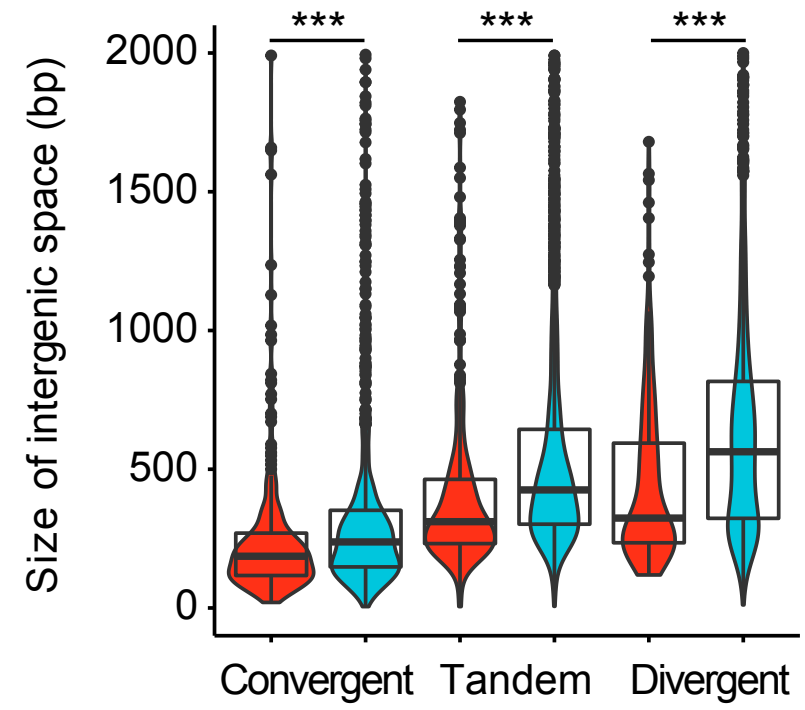

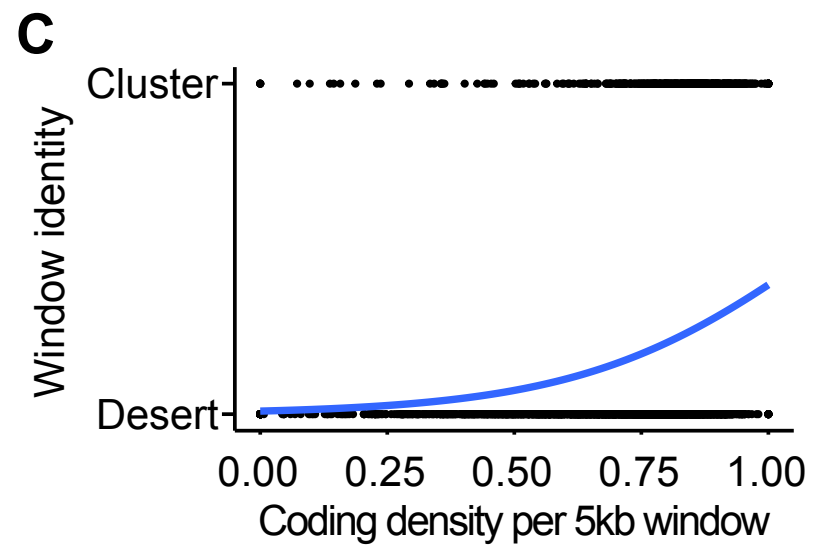
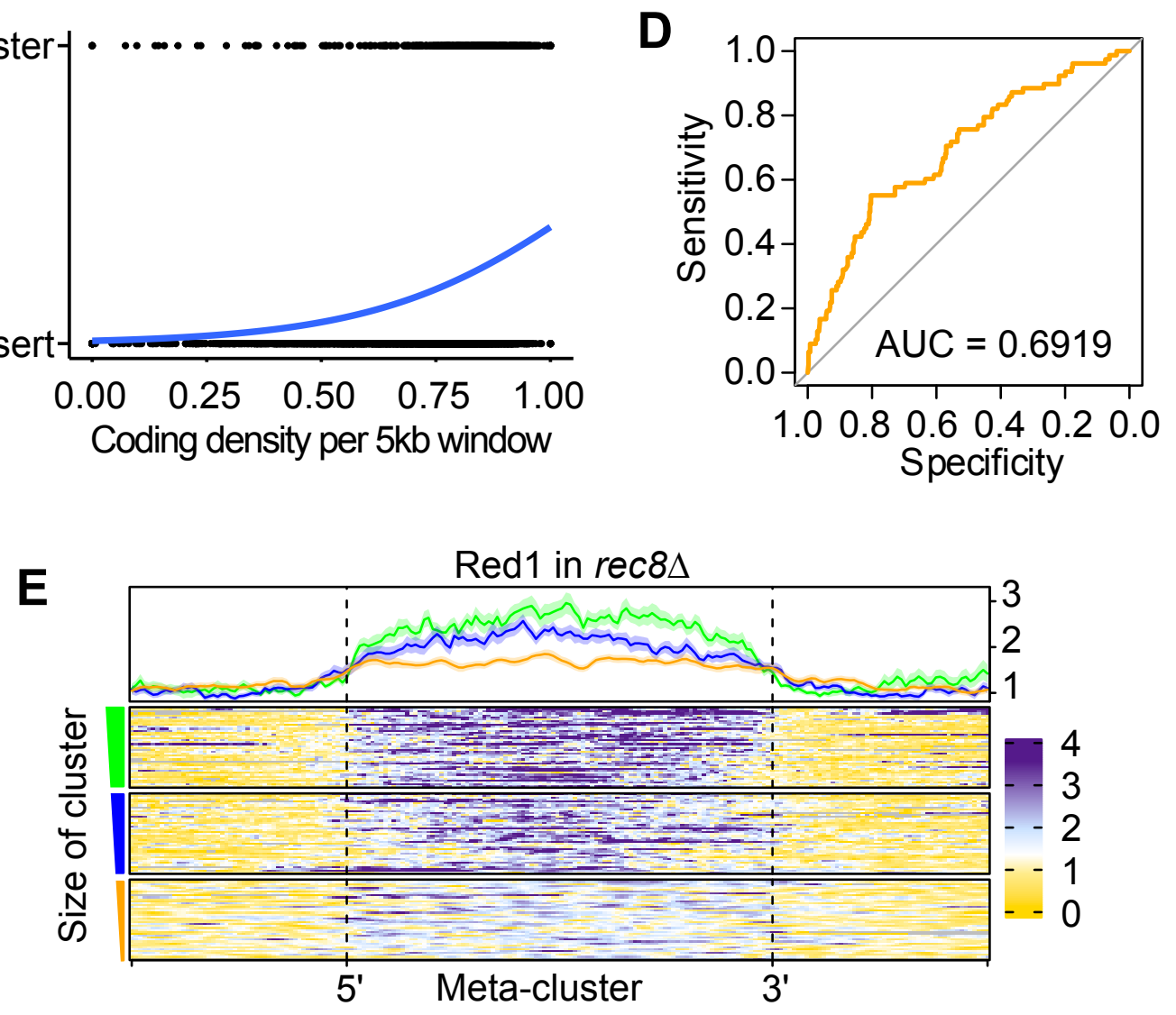

Figure 2 
Figure 2. Clusters are composed of long, closely spaced genes.

(A) The distribution of the gene sizes in clusters (red) and deserts (blue) displayed in violin and box plots. (B) The distribution of the sizes of convergent, tandem, and divergent regions in clusters (red) and deserts (blue). For (A-B), ${ }^{* * *} P<0.0001$, Mann-Whitney-Wilcoxon test. (C) Distribution of the coding density in 5-kb cluster windows and their identity assignment as clusters or deserts. The blue line indicates the fitted curve for the data. The coding density was chosen as a significant feature in training a logistic regression model $(P<0.0001)$ (D) ROC curve showing the specificity versus sensitivity of prediction of a subset of $20 \%$ of test data. The area under the curve (AUC) is 0.6919. (E) Meta analysis of Red1 ChIP-seq signal in rec8D in cluster regions sorted by size of the region (small = below $10 \mathrm{~kb}$; medium = $10 \mathrm{~kb}$ to $40 \mathrm{~kb}$; large $=40 \mathrm{~kb}$ to $210 \mathrm{~kb}$ ). Averages for each quantile are shown above the heatmap, and the $95 \%$ confidence interval for the average lines is shown. See also Figure S2. 
bioRxiv preprint doi: $h t t p s: / / d o i . o r g / 10.1101 / 2020.08 .11247122 \cdot$ this version posted August 12,2020 . The copyright holder for this preprint (which was not certified by peer review) is the author/funder, who has granted bioRxiv a license to display the preprint in perpetuity. It is made
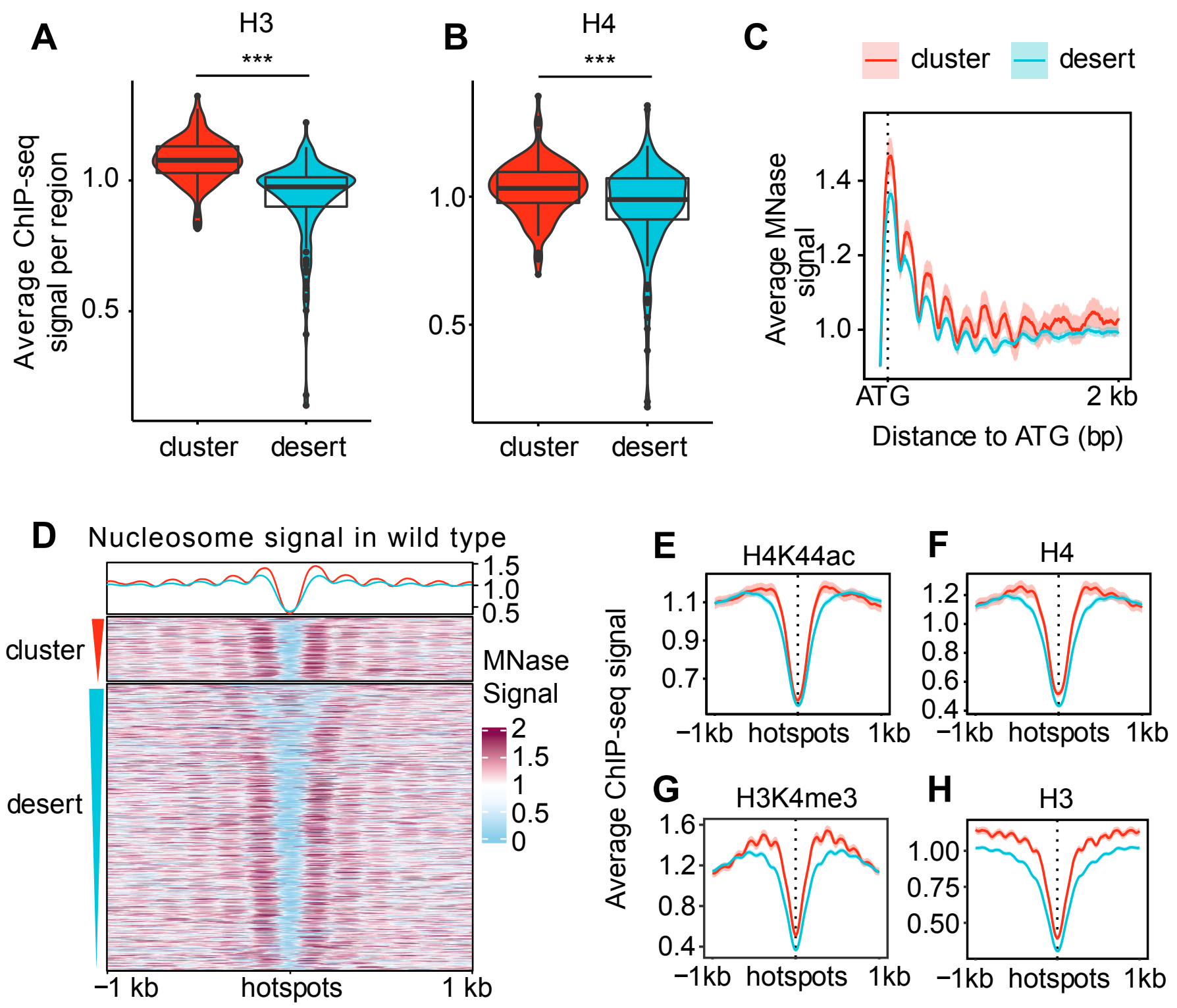
Figure 3. Hotspots in clusters are located in narrow intergenic regions surrounded by highly ordered nucleosomes.

Violin and box plots quantifying the average (A) $\mathrm{H} 3$ [23] and (B) $\mathrm{H} 4$ [24] signal per cluster or desert region on wild-type chromosomes at $4 \mathrm{~h}$ after meiotic induction. ${ }^{* * *} P<0.0001$, MannWhitney-Wilcoxon. (C) Average MNase signal for clusters (red) and deserts (blue) lined up at the ATG for every gene. (D) Heatmap of wild-type MNase signal centered at all hotspot midpoints [28], separated into cluster or desert regions and sorted by width of hotspots. The average signal in clusters and deserts is plotted above the heatmap. Average H4K44ac (E), H4 (F), H3K4me3 (G), and H3 (H) ChIP-seq signal lined up at hotspots in clusters and deserts. The $95 \%$ confidence interval for the average lines is shown for all averaged signals. See also Figure S3. 


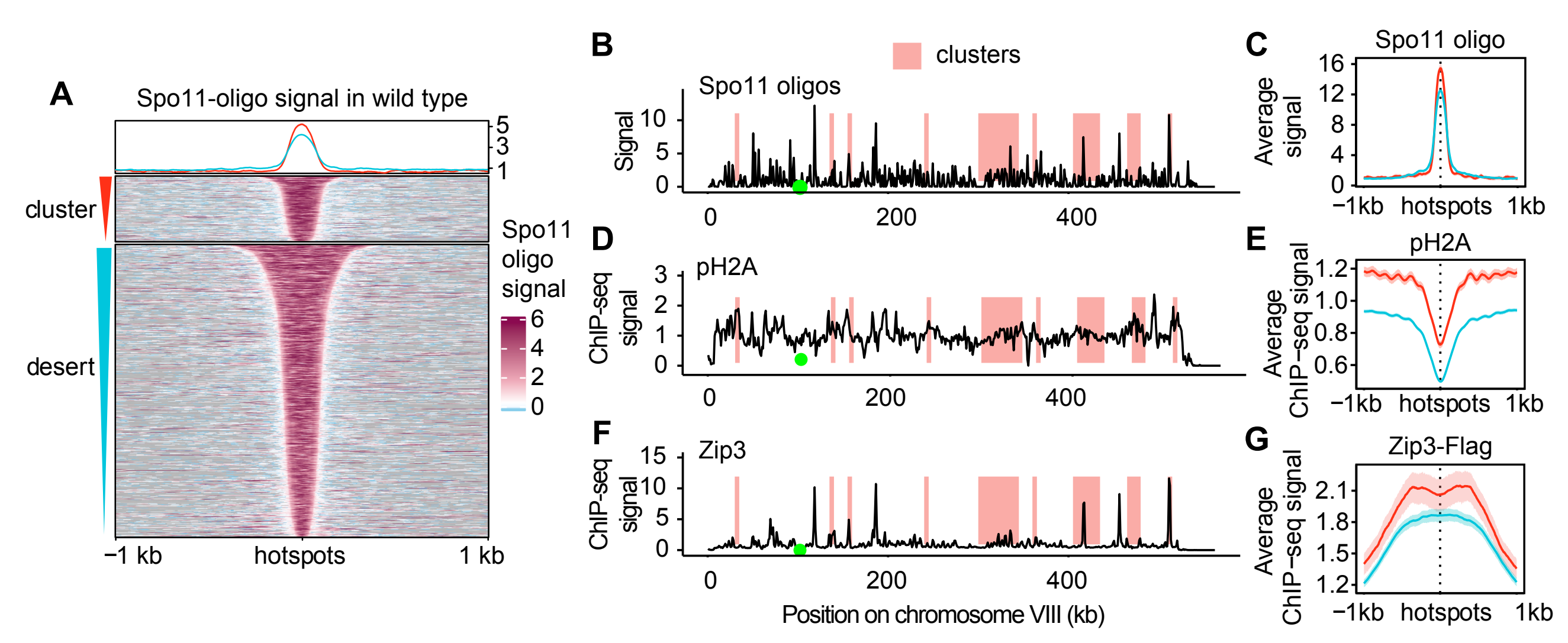

Figure 4 
Figure 4. DNA breakage and crossover designation are more frequent in clusters.

(A) Heatmap of Spo11-oligo signal [28] at 4h after meiotic induction centered at all hotspot

midpoints, separated into cluster or desert regions, and sorted by size of hotspots. The average

of signal in clusters (red) and deserts (blue) is plotted above the heatmap. Spo11-oligo signal

(B-C) and ChIP-seq analysis of pH2A at 3h after meiotic induction (D-E) and Zip3 at 4h after

meiotic induction [31] (F-G). The $\mathrm{pH} 2 \mathrm{~A}$ dataset was from an ndt80 $\Delta$ mutant, which is unable to exit meiotic prophase but shows no obvious defects at $3 \mathrm{~h}$ after meiotic induction [44]. (B, D, F)

Signal on wild-type chromosome VIII. Red underlay represents clusters, and green dot marks

centromere. (C, E, G) Average signal on wild-type chromosomes at cluster and desert hotspots.

The $95 \%$ confidence interval for the average lines is shown. See also Figure S4. 


\section{Materials and Methods}

\section{Yeast strains and growth conditions}

All strains used in this study were of the SK1 background, with the exception of the top2-1 mutant, which is congenic to SK1 (backcrossed >7x). The genotypes are listed in Table S1. To induce synchronous meiosis, strains were accumulated in $\mathrm{G} 1$ by inoculating BYTA medium with cells at OD600 $=0.3$ for 16.5 hours at $30^{\circ} \mathrm{C}$ [14]. Cultures were washed twice with water and resuspended into SPO medium at OD600 $=1.9-2.0$ at $30^{\circ} \mathrm{C}$ as described [14]. top2-1 cells were inoculated at OD600 $=0.8$ in BYTA medium for 20 hours at room temperature. For experiments that included top2-1 mutants, SPO cultures for all strains were washed twice with water and resuspended into SPO medium at OD600 = 1.9 at room temperature and shifted to $34^{\circ} \mathrm{C}$ after 1 hour. For the Brn1-FRB anchor away experiment, rapamycin was added to a final concentration of $1 \mu \mathrm{M}$ at the time of meiotic induction $(0 \mathrm{~h})$.

\section{Chromosome spreads and immunofluorescence}

Meiotic nuclear spreads were performed as described [45]. Red1 was detected using anti-Red1 rabbit serum (Lot\#16441; kind gift of N. Hollingsworth) at 1:100 and Alexa Fluor 488 anti-rabbit antibody at 1:100. Microscopy and image processing were carried out using a Deltavision Elite imaging system (Applied Precision) adapted to an Olympus IX17 microscope and analyzed using softWoRx 5.0 software.

\section{Chromatin immunoprecipitation}

At the indicated time points ( 0 or $3 \mathrm{~h}$ ), $25 \mathrm{ml}$ of meiotic culture was harvested and fixed for $30 \mathrm{~min}$ in $1 \%$ formaldehyde. Formaldehyde was quenched by addition of $125 \mathrm{mM}$ glycine and samples processed as described [46]. Samples were immunoprecipitated with $2 \mu \mathrm{L}$ of either anti-H3 (Abcam ab1791), anti-Hop1 (kind gift of $\mathrm{N}$. Hollingsworth) or anti-Red1 (kind gift of $\mathrm{N}$. Hollingsworth, \#16440) per IP. Library preparation was completed as described [7]. Library quality 
was confirmed by Qubit HS assay kit and either Agilent 2100 Bioanalyzer or 2200 TapeStation. $50 \mathrm{bp}$ or $51 \mathrm{bp}$ single-end sequencing was accomplished on an Illumina HiSeq 2500 or NextSeq 500 instrument. Read length and sequencing instrument did not introduce any obvious biases to the results.

\section{Mononucleosomal DNA preparation}

At the 0 - or 3-hr time point, $50 \mathrm{ml}$ of meiotic culture was harvested and fixed for $30 \mathrm{~min}$ in $1 \%$ formaldehyde. The formaldehyde was quenched by addition of $125 \mathrm{mM}$ glycine and samples processed as described [25]. Library preparation and sequencing were done as outlined under the chromatin immunoprecipitation section above.

\section{Processing of Illumina sequence data}

Sequencing reads were mapped to the SK1 genome [47] using Bowtie. Sequencing reads of 100 bp were clipped to $51 \mathrm{bp}$. Only perfect matches across all $51 \mathrm{bp}$ were considered during mapping. Multiple alignments were not taken into account, which means each read only mapped to one location in the genome. Reads were extended towards 3' ends to a final length of 200 bp and probabilistically determined PCR duplications were removed in MACS-2.1.1 (https://github.com/taoliu/MACS) [48]. All pileups were SPMR-normalized (signal per million reads), and fold-enrichment of the ChIP data over the input data was calculated. Plots shown were made using two or more combined replicates. The $95 \%$ confidence intervals were calculated by bootstrap resampling from the data 1000 times with replacement.

\section{Peak Calling}

To identify Red1, Hop1, and Rec8 protein enriched regions (peaks) for Figure 1E, MACS-2.1.1 (https://github.com/taoliu/MACS) [48] was used for peak calling of the sequence data. The reads were processed identically to the description in the "Processing of Illumina sequence data," except 
the --broad flag was used with the "callpeak" function to determine significant regions of enrichment that meet the default q-value cutoff.

\section{Defining clusters and deserts}

The Red1 ChIP-seq sequencing data in the rec8 $\Delta$ mutant was partitioned into 5000 bp bins with signal scores equal to the average signal across the bin. Bins with scores greater than or equal to 1.75 times the standard deviation of the Red 1 signal across the genome were classified as enriched regions. Enriched regions were joined with adjacent enriched regions to define "clusters". The remaining regions were defined as "deserts".

\section{Logistic regression modeling}

The genome was divided into 5000 bp bins, matching the bins used to define clusters. Each bin was classified as either a cluster or a desert and the coding density was calculated for each bin. The caret package (https://github.com/topepo/caret/) was used to create a training set of $80 \%$ of the data and a test set of the remaining $20 \%$ of the data, and to train a logistic regression model using the training data. The coding density was determined to be a significant feature $(P<0.0001)$. This model was applied to the test data to predict the cluster status using the input coding density.

\section{Data Availability}

The datasets and computer code produced in this study are available in following databases:

- ChIP-seq data data: Gene Expression Omnibus GSE156040

$$
\text { 'https://www.ncbi.nlm.nih.gov/geo/query/acc.cgi?acc=GSE156040’ }
$$

- Computer scripts for processing Illumina reads: Github

'https://github.com/hochwagenlab/ChIPseq functions/tree/master/ChIPseq Pipeline v3/'

Computer scripts for making figures: Github "https://github.com/hochwagenlab/axis clusters " 


\section{$\underline{\text { References }}$}

1. Borde, V., and de Massy, B. (2013). Programmed induction of DNA double strand breaks during meiosis: Setting up communication between DNA and the chromosome structure. Current Opinion in Genetics and Development 23, 147-155.

2. Lam, I., and Keeney, S. (2015). Mechanism and regulation of meiotic recombination initiation. Cold Spring Harbor Perspectives in Biology 7.

3. Székvölgyi, L., Ohta, K., and Nicolas, A. (2015). Initiation of meiotic homologous recombination: Flexibility, impact of histone modifications, and chromatin remodeling. Cold Spring Harbor Perspectives in Biology 7, 1-18.

4. Zickler, D., and Kleckner, N. (2015). Recombination, pairing, and synapsis of homologs during meiosis. Cold Spring Harbor Perspectives in Biology 7, 1-28.

5. Page, S.L., and Hawley, R.S. (2004). THE GENETICS AND MOLECULAR BIOLOGY OF THE SYNAPTONEMAL COMPLEX. Annual Review of Cell and Developmental Biology 20, 525-558. Available at: www.annualreviews.org [Accessed October 1, 2019].

6. Panizza, S., Mendoza, M.A., Berlinger, M., Huang, L., Nicolas, A., Shirahige, K., and Klein, F. (2011). Spol1-accessory proteins link double-strand break sites to the chromosome axis in early meiotic recombination. Cell 146, 372-383.

7. Sun, X., Huang, L., Markowitz, T.E., Blitzblau, H.G., Chen, D., Klein, F., and Hochwagen, A. (2015). Transcription dynamically patterns the meiotic chromosome-axis interface. eLife 4, e07424. Available at: http://www.ncbi.nlm.nih.gov/pubmed/26258962.

8. Kugou, K., Fukuda, T., Yamada, S., Ito, M., Sasanuma, H., Mori, S., Katou, Y., Itoh, T., Matsumoto, K., Shibata, T., et al. (2009). Rec8 guides canonical Spo11 distribution along yeast meiotic chromosomes. Mol Biol Cell 20, 3064-3076.

9. Klein, F., Mahr, P., Galova, M., Buonomo, S.B.C., Michaelis, C., Nairz, K., and Nasmyth, K. (1999). A central role for cohesins in sister chromatid cohesion, formation of axial elements, and recombination during yeast meiosis. Cell 98, 91-103.

10. Schalbetter, S.A., Fudenberg, G., Baxter, J., Pollard, K.S., and Neale, M.J. (2019). Principles of meiotic chromosome assembly revealed in S. cerevisiae. Nature Communications 10.

11. Vale-Silva, L.A., Markowitz, T.E., and Hochwagen, A. (2019). SNP-ChIP: A versatile and tag-free method to quantify changes in protein binding across the genome. BMC Genomics 20, 54. Available at: http://www.ncbi.nlm.nih.gov/pubmed/30654749.

12. Lambing, C., Tock, A.J., Topp, S.D., Choi, K., Kuo, P.C., Zhao, X., Osman, K., Higgins, J.D., Franklin, F.C.H., and Henderson, I.R. (2020). Interacting Genomic Landscapes of REC8-Cohesin, Chromatin, and Meiotic Recombination in Arabidopsis. The Plant cell.

13. Markowitz, T.E., Heldrich, J., and Hochwagen, A. (2020). DNA break formation induces Scc2/cohesin-dependent recruitment of condensin to meiotic chromosomes. bioRxiv 11, 23-29.

14. Falk, J.E., Chan, A.C. ho, Hoffmann, E., and Hochwagen, A. (2010). A Mec1- and PP4Dependent checkpoint couples centromere pairing to meiotic recombination.

Developmental Cell 19, 599-611.

15. Heldrich, J., Sun, X., Vale-Silva, L.A., Markowitz, T.E., and Hochwagen, A. (2020). Topoisomerases Modulate the Timing of Meiotic DNA Breakage and Chromosome Morphogenesis in Saccharomyces cerevisiae. Genetics. 
16. Haruki, H., Nishikawa, J., and Laemmli, U.K. (2008). The Anchor-Away Technique: Rapid, Conditional Establishment of Yeast Mutant Phenotypes. Molecular Cell 31, 925932. Available at: http://www.ncbi.nlm.nih.gov/pubmed/18922474.

17. Paul, M., Markowitz, T.E., Hochwagen, A., and Ercan, S. (2018). Condensin Depletion Causes Genome Decompaction Without Altering the Level of Global Gene Expression in Saccharomyces cerevisiae. Genetics 210, 331-344. Available at: http://www.ncbi.nlm.nih.gov/pubmed/29970489.

18. DiNardo, S., Voelkel, K., and Sternglanz, R. (1984). DNA topoisomerase II mutant of Saccharomyces cerevisiae: Topoisomerase II is required for segregation of daughter molecules at the termination of DNA replication. Proceedings of the National Academy of Sciences of the United States of America 81, 2616-2620. Available at: http://www.ncbi.nlm.nih.gov/pubmed/6326134.

19. Blitzblau, H.G., Bell, G.W., Rodriguez, J., Bell, S.P., and Hochwagen, A. (2007). Mapping of Meiotic Single-Stranded DNA Reveals Double-Strand-Break Hotspots near Centromeres and Telomeres. Current Biology 17, 2003-2012. Available at: http://www.ncbi.nlm.nih.gov/pubmed/18060788.

20. Subramanian, V. v, Zhu, X., Markowitz, T.E., Vale-Silva, L.A., San-Segundo, P.A., Hollingsworth, N.M., Keeney, S., and Hochwagen, A. (2019). Persistent DNA-break potential near telomeres increases initiation of meiotic recombination on short chromosomes. Nature Communications 10, 970. Available at: http://www.ncbi.nlm.nih.gov/pubmed/30814509.

21. Murakami, H., Lam, I., Huang, P.C., Song, J., van Overbeek, M., and Keeney, S. (2020). Multilayered mechanisms ensure that short chromosomes recombine in meiosis. Nature 582, 124-128.

22. Brogaard, K., Xi, L., Wang, J.P., and Widom, J. (2012). A map of nucleosome positions in yeast at base-pair resolution. Nature 486, 496-501.

23. Zhu, X., and Keeney, S. (2015). High-resolution global analysis of the influences of Bas1 and Ino4 transcription factors on meiotic DNA break distributions in saccharomyces cerevisiae. Genetics 201, 525-542. Available at: http://www.ncbi.nlm.nih.gov/pubmed/26245832.

24. Hu, J., Donahue, G., Dorsey, J., Govin, J., Yuan, Z., Garcia, B.A., Shah, P.P., and Berger, S.L. (2015). H4K44 Acetylation Facilitates Chromatin Accessibility during Meiosis. Cell Reports 13, 1772-80.

25. Pan, J., Sasaki, M., Kniewel, R., Murakami, H., Blitzblau, H.G., Tischfield, S.E., Zhu, X., Neale, M.J., Jasin, M., Socci, N.D., et al. (2011). A hierarchical combination of factors shapes the genome-wide topography of yeast meiotic recombination initiation. Cell 144, 719-731.

26. Cucinotta, C.E., Hildreth, A.E., McShane, B.M., Shirra, M.K., and Arndt, K.M. (2019). The nucleosome acidic patch directly interacts with subunits of the Pafl and FACT complexes and controls chromatin architecture in vivo. Nucleic acids research 47, 84108423.

27. Gothwal, S.K., Patel, N.J., Colletti, M.M., Sasanuma, H., Shinohara, M., Hochwagen, A., and Shinohara, A. (2016). The double-strand break landscape of meiotic chromosomes is shaped by the Paf1 transcription elongation complex in Saccharomyces cerevisiae. Genetics 202, 497-512. Available at: http://www.ncbi.nlm.nih.gov/pubmed/26627841. 
28. Thacker, D., Mohibullah, N., Zhu, X., and Keeney, S. (2014). Homologue engagement controls meiotic DNA break number and distribution. Nature 510, 241-246. Available at: http://www.ncbi.nlm.nih.gov/pubmed/24717437.

29. Chen, S.Y., Tsubouchi, T., Rockmill, B., Sandler, J.S., Richards, D.R., Vader, G., Hochwagen, A., Roeder, G.S., and Fung, J.C. (2008). Global Analysis of the Meiotic Crossover Landscape. Developmental Cell 15, 401-415. Available at:

http://www.ncbi.nlm.nih.gov/pubmed/18691940.

30. Zhang, L., Wang, S., Yin, S., Hong, S., Kim, K.P., and Kleckner, N. (2014).

Topoisomerase II mediates meiotic crossover interference. Nature 511, 551-556.

Available at: http://www.ncbi.nlm.nih.gov/pubmed/25043020.

31. Serrentino, M.E., Chaplais, E., Sommermeyer, V., and Borde, V. (2013). Differential Association of the Conserved SUMO Ligase Zip3 with Meiotic Double-Strand Break Sites Reveals Regional Variations in the Outcome of Meiotic Recombination. PLoS Genetics 9, e1003416. Available at: https://dx.plos.org/10.1371/journal.pgen.1003416 [Accessed June 1, 2020].

32. Joshi, N., Barot, A., Jamison, C., and Börner, G.V. (2009). Pch2 links chromosome axis remodeling at future crossover sites and crossover distribution during yeast meiosis. PLoS Genetics 5, 1000557. Available at: www.plosgenetics.org [Accessed October 15, 2019].

33. Börner, G.V., Barot, A., and Kleckner, N. (2008). Yeast Pch2 promotes domainal axis organization, timely recombination progression, and arrest of defective recombinosomes during meiosis. Proceedings of the National Academy of Sciences of the United States of America 105, 3327-3332. Available at: http://www.ncbi.nlm.nih.gov/pubmed/18305165.

34. Kim, K.P., Weiner, B.M., Zhang, L., Jordan, A., Dekker, J., and Kleckner, N. (2010). Sister cohesion and structural axis components mediate homolog bias of meiotic recombination. Cell 143, 924-937.

35. Yatskevich, S., Rhodes, J., and Nasmyth, K. (2019). Organization of Chromosomal DNA by SMC Complexes. Annu Rev Genet 53, 445-482. Available at: http://www.ncbi.nlm.nih.gov/pubmed/31577909.

36. Uhlmann, F. (2016). SMC complexes: from DNA to chromosomes. Nat Rev Mol Cell Biol 17, 399-412. Available at: http://www.ncbi.nlm.nih.gov/pubmed/27075410.

37. Wang, J.C. (2002). Cellular roles of DNA topoisomerases: A molecular perspective. Nature Reviews Molecular Cell Biology 3, 430-440. Available at:

http://www.ncbi.nlm.nih.gov/pubmed/12042765.

38. Pommier, Y., Sun, Y., Huang, S.Y.N., and Nitiss, J.L. (2016). Roles of eukaryotic topoisomerases in transcription, replication and genomic stability. Nature Reviews Molecular Cell Biology 17, 703-721.

39. Primig, M., Williams, R.M., Winzeler, E.A., Tevzadze, G.G., Conway, A.R., Hwang, S.Y., Davis, R.W., and Esposito, R.E. (2000). The core meiotic transcriptome in budding yeasts. Nature Genetics 26, 415-423.

40. Chu, S., DeRisi, J., Eisen, M., Mulholland, J., Botstein, D., Brown, P.O., and Herskowitz, I. (1998). The transcriptional program of sporulation in budding yeast. Science 282, 699-705.

41. Kironmai, K.M., Muniyappa, K., Friedman, D.B., Hollingsworth, N.M., and Byers, B. (1998). DNA-Binding Activities of Hop1 Protein, a Synaptonemal Complex Component from Saccharomyces cerevisiae. Molecular and Cellular Biology 18, 1424-1435. 
42. Kshirsagar, R., Khan, K., Joshi, M. v., Hosur, R. v., and Muniyappa, K. (2017). Probing the Potential Role of Non-B DNA Structures at Yeast Meiosis-Specific DNA DoubleStrand Breaks. Biophysical Journal 112, 2056-2074.

43. Agarwal, S., and Roeder, G.S. (2000). Zip3 provides a link between recombination enzymes and synaptonemal complex proteins. Cell 102, 245-255.

44. Subramanian, V. v., MacQueen, A.J., Vader, G., Shinohara, M., Sanchez, A., Borde, V., Shinohara, A., and Hochwagen, A. (2016). Chromosome Synapsis Alleviates Mek1Dependent Suppression of Meiotic DNA Repair. PLoS Biology 14, e1002369. Available at: http://www.ncbi.nlm.nih.gov/pubmed/26870961 [Accessed September 26, 2019].

45. Subramanian, V. v, MacQueen, A.J., Vader, G., Shinohara, M., Sanchez, A., Borde, V., Shinohara, A., and Hochwagen, A. (2016). Chromosome Synapsis Alleviates Mek1Dependent Suppression of Meiotic DNA Repair. PLoS Biology 14, e1002369. Available at: http://www.ncbi.nlm.nih.gov/pubmed/26870961.

46. Blitzblau, H.G., and Hochwagen, A. (2013). ATR/Mec1 prevents lethal meiotic recombination initiation on partially replicated chromosomes in budding yeast. eLife 2, e00844. Available at: http://www.ncbi.nlm.nih.gov/pubmed/24137535.

47. Yue, J.X., Li, J., Aigrain, L., Hallin, J., Persson, K., Oliver, K., Bergström, A., Coupland, P., Warringer, J., Lagomarsino, M.C., et al. (2017). Contrasting evolutionary genome dynamics between domesticated and wild yeasts. Nature Genetics 49, 913-924. Available at: http://www.ncbi.nlm.nih.gov/pubmed/28416820.

48. Zhang, Y., Liu, T., Meyer, C.A., Eeckhoute, J., Johnson, D.S., Bernstein, B.E., Nussbaum, C., Myers, R.M., Brown, M., Li, W., et al. (2008). Model-based analysis of ChIP-Seq (MACS). Genome Biology 9, R137. Available at: http://www.ncbi.nlm.nih.gov/pubmed/18798982. 